\title{
Best constant in Sobolev trace inequalities on the half-space
}

\author{
Bruno Nazaret*
}

April 2005

\begin{abstract}
Using a mass transportation method, we study optimal Sobolev trace inequalities on the half-space and prove a conjecture made by J.F. Escobar in 1988 about the minimizers.
\end{abstract}

\section{Introduction}

Let $n \geq 3$ be an integer and $p$ a real in $(1, n)$. The classical Sobolev inequality in $\mathbb{R}^{n}$ asserts the existence of an universal positive constant $K_{n}(p)$, such that, for all function $f$ in the Sobolev space $\mathrm{W}^{1, p}\left(\mathbb{R}^{n}\right)$,

$$
\|f\|_{L^{p^{*}}\left(\mathbb{R}^{n}\right)} \leq K_{n}(p)\|\nabla f\|_{L^{p}\left(\mathbb{R}^{n}\right)}
$$

with $p^{*}=\frac{n p}{n-p}$.

In fact, the space $\mathrm{W}^{1, p}$ is not well adapted to study Sobolev inequalities on $\mathbb{R}^{n}$, and should be replaced by the homogeneous Sobolev space

$$
\dot{\mathrm{W}}^{1, p}\left(\mathbb{R}^{n}\right)=\left\{f \in L^{p^{*}}\left(\mathbb{R}^{n}\right) \text {, s. t. } \nabla f \in L^{p}\left(\mathbb{R}^{n}\right)\right\},
$$

or equivalently (see [23]), the space of functions vanishing at infinity with their gradient in $L^{p}\left(\mathbb{R}^{n}\right)$. We will say that a measurable function $f: \mathbb{R}^{n} \rightarrow \mathbb{R}$ vanishes at infinity if

$$
\forall a>0, \quad \operatorname{mes}\left(\left\{x \in \mathbb{R}^{n} ;|f(x)| \geq a\right\}\right)<+\infty .
$$

The best constant in (1), together with extremal functions, was found in 1974 independently by Aubin [2] and Talenti [24] by classical variational methods. By a symmetrization argument, the problem is reduced to the dimension one, and then solved by ODE techniques. The extremal functions, up to dilations and translations, take then the following form,

$$
f(y)=\left(\epsilon^{p}+|y|^{p}\right)^{1-\frac{n}{p}}, \quad \epsilon>0 .
$$

Consider now a smooth bounded domain $\Omega \subset \mathbb{R}^{n}$. The inequality (1) does not hold anymore, because of the constant functions. Then, a natural strategy is to replace the condition (2) on the functions by a vanishing condition on the boundary, that is to work on the space $\mathrm{W}_{0}^{1, p}(\Omega)$. In that setting, Guedda and Veron proved in [17] that, for a bounded

\footnotetext{
*Ceremade - Université de Paris-Pauphine, Place du Maréchal Delattre de Tassigny, 75775 Paris Cedex 16, France
} 
and starpshaped domain $\Omega$, the corresponding inequality does not admit extremal function. In [13], F. Demengel and E. Hebey studied a modified version:

$$
I_{p}(\Omega)=\inf _{u \in \mathrm{W}_{0}^{1, p}\left(\mathbb{R}^{n}\right), \int_{\Omega} f(x)|u(x)|^{p^{*}} d x=1}\left(\int_{\Omega}\left(|\nabla u(x)|^{p}+a(x)|u(x)|^{p}\right) d x\right) .
$$

They proved that the existence of extremal functions to (4) is related to the following condition

$$
I_{p}(\Omega) K_{n}(p)^{p}\left(\max _{x \in \Omega} f(x)\right)^{1-\frac{n}{p}}<1 .
$$

The value of $I_{p}(\Omega)$ being unknown, they use the extremals in (1) in order to get explicit conditions on the domain and the data $a, f$, by localization around a point of maximum of $f$. A generalization of (4) by Demengel and the author leads to the subject of the present paper. Replacing the constraint in (4) by

$$
u \in \mathrm{W}^{1, p}(\Omega) ; \quad \int_{\partial \Omega} f(x)|u(x)|^{\tilde{p}} d x=1,
$$

where $\tilde{p}=\frac{(n-1) p}{n-p}$ is the critical exponent for Sobolev imbeddings into trace spaces, an existence condition similar to (5) is found, the reference constant $K_{n}(p)$ being replaced by the best constant in the following Sobolev inequality on $\mathbb{R}_{+}^{n}=\mathbb{R}_{+} \times \mathbb{R}^{n-1}$,

$$
\|f\|_{L^{\tilde{p}}\left(\partial \mathbb{R}_{+}^{n}\right)} \leq Q_{n}(p)\|\nabla f\|_{L^{p}\left(\mathbb{R}_{+}^{n}\right)} .
$$

If we know the extremals in (6), the work done in [13] can be adapted to get explicit existence conditions of minimizers. Notice that the existence of extremals for (6) has been established by Lions in [19] using the concentration-compactness principle. In the case where $p=2$, these extremals (and then the best constant) have been computed by Escobar in [15], and further by Beckner in [4]. Unfortunately, their proofs both deeply used the conformal invariance of the associated variational problem, then cannot be generalized to other values of $p$ and the problem was still open. In his paper, Escobar conjectured that the functions

$$
\forall(t, x) \in \mathbb{R}_{+}^{n}, \quad f_{\lambda}(t, x)=\frac{1}{\left((t+\lambda)^{2}+|x|^{2}\right)^{\frac{n-p}{2(p-1)}}}, \quad \lambda>0
$$

are optimal in (6). This is natural since they solve the PDE

$$
\begin{cases}-\operatorname{div}\left(|\nabla f|^{p-2} \nabla f\right)=0 & \text { in } \mathbb{R}_{+}^{n} \\ |\nabla f|^{p-2} \nabla f \cdot \mathbf{n}=-Q_{n}^{\prime}(p) f^{\frac{n(p-1)}{n-p}} & \text { on } \partial \mathbb{R}_{+}^{n} .\end{cases}
$$

The difficulty is to prove that $Q_{n}^{\prime}(p)=Q_{n}(p)^{-p}$. It is impossible to reduce the problem to the dimension 1 , since symmetrization arguments only apply with respect to the $x$ variable, and don't really simplify it (see [18] for such a construction). The main result of this paper can be stated as follows.

Theorem 1. Let $n \geq 3$ and $p$ a real in $(1, n)$. Let $\|\cdot\|$ be a norm on $\mathbb{R}^{n}$, its dual norm being denoted by $\|\cdot\|_{*}$. Then, for all $f$ in $\dot{\mathrm{W}}^{1, p}\left(\mathbb{R}_{+}^{n}\right)$,

$$
\|f\|_{L^{\frac{(n-1) p}{n-p}}\left(\partial \mathbb{R}_{+}^{n}\right)} \leq Q_{n}(p)\left(\int_{\mathbb{R}_{+}^{n}}\|\nabla f(y)\|_{*}^{p} d y\right)^{\frac{1}{p}},
$$


with equality in (8) as soon as, for some $\lambda>0$ and $x_{0} \in \mathbb{R}^{n-1}$,

$$
\forall(t, x) \in \mathbb{R}_{+}^{n}, \quad f(t, x)=\left(\frac{\lambda^{\frac{1}{p}}}{\left\|\left(t+\lambda, x-x_{0}\right)\right\|}\right)^{\frac{n-p}{(p-1)}} .
$$

It contains the conjecture of [15], in the particular case of the Euclidean norm. Using the particular form of the expected extremals, the proof of this result uses a mass transportation method, and is deeply inspired by [11] and more recently [20]. In [20], Maggi and Villani proved an optimal inequality valid on all locally Lipschitz domains $\Omega$ :

$$
\|f\|_{L^{p^{*}}(\Omega)} \leq K_{n}(p)\|\nabla f\|_{L^{p}(\Omega)}+T_{n}(p)^{-1}\|f\|_{L^{\tilde{p}}(\partial \Omega)},
$$

where $\tilde{p}=\frac{(n-1) p}{n-p}$ (this exponent is the critical ones for the Sobolev embedding into $L^{q}$ space on the boundary), and $T_{n}(p)^{-\tilde{p}}$ is the isoperimetric constant. In addition, they showed that (10) is sharp on balls. This generalizes in particular a result of Brezis and Lieb in [6], correponding to $p=2$.

The results of [20] stand in the continuation of the considerable efforts which have been spent in the last years to get a better understanding of Sobolev type inequalities, with the introduction of mass transportation concepts in the field. These methods are not new and, for example, are somehow used in all known proofs of the Brünn-Minkowski inequality:

$$
|A+B|^{\frac{1}{n}} \geq|A|^{\frac{1}{n}}+|B|^{\frac{1}{n}}
$$

for all measurable sets $A$ and $B$ in $\mathbb{R}^{n}$. In the appendix of [22], Gromov gave a proof of the isoperimetric inequality based on the Knothe transport map between two probability densities. After the work of Brenier on the polar factorization [5] and more especially of McCann on the Monge-Kantorovitch optimal transportation problem [21], Otto and Villani found deep links between log-Sobolev inequality and the asymptotic profiles in the FokkerPlanck equation. In [12], Dolbeault and Del Pino used the entropy-entropy production method to prove that asymptotics in nonlinear diffusion equations were exactly the extremals of a one-parameter family of Gagliardo-Nirenberg inequalities, with (1) as a limit case, and obtained these extremals by similar methods as Aubin and Talenti. Inspired on the one hand by this work, and on the other hand by the direct proof using a mass transportation method of Gaussian inequalities obtained by Barthe in [3], Cordero-Erausquin, Villani and the author exhibited in [11] a completely new proof of (1) in its optimal form (together with the whole family of Gagliardo-Nirenberg inequalities of [12]). This result is derived from a new duality principle generalized later by Agueh, Ghoussoub and Kang in [1]. The proof of this duality principle is direct, by writing a sequence of elementary inequalities, each of them being optimal. The extremals are then recovered by tracing back the cases of equality at each step. In addition, except from the Brenier map, it only involves arithmetic-geometric and Hölder inequalities. For that reason, the result is obtained for an arbitrary norm on $\mathbb{R}^{n}$, and it is possible to prove that, up to translations and dilations, the functions given by (3) are the only minimizers in (1), avoiding the use of the moving plane method [16], or competting symmetries of Carlen and Loss [10]. Contrary to the inequalities covered by [11], which have already been proved by variational approach, the inequalities studied in [20] and this work have no other proof. This shows that mass transportation techniques are particularly adapted to inequalities involving trace terms. Actually, since the proof applies for any norm on $\mathbb{R}_{+}^{n}$, it generalizes in the case $p=2$ the result of [15] and [4], showing that neither conformal invariance nor the Euclidean structure of $\mathbb{R}^{n}$ play any role in the problem of sharp Sobolev inequalities with trace terms. 
The next section is devoted to the proof of theorem 1, after a summary of the mass transportation ingredients and some basic considerations about norms on Euclidean spaces. In the last section, we shall give some comments about uniqueness and results which can be derived from the proof, especially when interesting to arbitrary domains.

\section{Proof of theorem 1}

First, we give a brief presentation of the key ingredients of the analysis, and recall the main properties of the Brenier map. Let $F$ and $G$ some probability densities on $\mathbb{R}_{+}^{n}$. By a result of Brenier [5], further refined by McCann [21], there exists a function $T=\nabla \varphi: \mathbb{R}_{+}^{n} \rightarrow \mathbb{R}_{+}^{n}$, with $\varphi$ convex, such that $T$ maps the measure $F(y) d y$ onto $G(y) d y$, which means by definition that, for all measurable set $B$,

$$
\int_{B} G(y) d y=\int_{T^{-1}(B)} F(y) d y,
$$

or, equivalently, for all measurable function $\psi: \mathbb{R}_{+}^{n} \rightarrow \mathbb{R}$,

$$
\int_{\mathbb{R}_{+}^{n}} \psi(y) G(y) d y=\int_{\mathbb{R}_{+}^{n}} \psi(T(y)) F(y) d y .
$$

With this properties, $T$ is uniquely determined $F$-a.e. Note, even if it is not crucial in this work, that $T$ realizes the optimal transportation in the Monge-Kantorovich problem with quadratic cost (see [25] for a very complete review on this subject) from $F(y) d y$ to $G(y) d y$. Assuming that the function $T$ is $C^{1}$ and realizes a diffeomorphism, which is in general completely unrealistic, a change of variables in (13) leads to the following Monge-Ampère equation:

$$
F(y)=G(\nabla \varphi(y)) \operatorname{Det} D^{2} \varphi(y) .
$$

In the general setting of probability densities, McCann proved in [21] that (14) remains valid at least $F$ almost everywhere, $D^{2} \varphi$ being undestood as the absolutely continuous part of the of the distributional Hessian matrix of $\varphi$ (or in the Alexandrov sense, that is, the quadratic part obtained in a second order Taylor expansion). The only more general regularity result known is due to Caffarelli $[7,8,9]$ for $C^{0, \alpha}$ densities with compact convex support, in which case the potential $\varphi$ becomes of classe $C^{2, \alpha}$ and then equation (14) holds in the classical sense. Note, as done in [20], that if the arrival density $G$ is compactly supported in $\mathbb{R}_{+}^{n}$, then the potential $\varphi$ can be assumed to have its domain the whole $\mathbb{R}^{n}$, since it is possible to extend it as a convex function outside $\mathbb{R}_{+}^{n}$. The main consequence of this fact is that we can assume that $\nabla \varphi$ has bounded variations up to the boundary.

Now, we introduce the underlying structure. Consider the vector space $\mathbb{R}^{n}$ endowed with some arbitrary norm denoted by $\|\cdot\|$. Then, the dual norm on $\left(\mathbb{R}^{n}\right)^{*}=\mathbb{R}^{n}$ is defined for all $X \in \mathbb{R}^{n}$ by

$$
\|X\|_{*}=\sup _{\|Y\|=1} X \cdot Y .
$$

It means that $\|\cdot\|_{*}$ is the conjugate function of the convex function $\|\cdot\|$. With this setting, the Hölder inequality holds: $\forall f:\left(\mathbb{R}^{n}\right)^{*} \rightarrow \mathbb{R}$ in $L^{p}$, and $\forall g: \mathbb{R}^{n} \rightarrow \mathbb{R}$ in $L^{q}$, with $\frac{1}{p}+\frac{1}{q}=1$,

$$
\int_{\mathbb{R}^{n}} f \cdot g \leq\left(\int_{\mathbb{R}^{n}}\|f\|_{*}^{p}\right)^{\frac{1}{p}}\left(\int_{\mathbb{R}^{n}}\|g\|^{q}\right)^{\frac{1}{q}},
$$


which expresses the fact that $L^{p}\left(\left(\mathbb{R}^{n}\right)^{*},\|\cdot\|_{*}\right)$ is the dual space of $L^{q}\left(\mathbb{R}^{n},\|\cdot\|\right)$. In addition, the norm function is differentiable at all $x \in \mathbb{R}^{n}, x \neq 0$, and for all such $x, \nabla(\|\cdot\|)(x)$ is the unique vector $x^{*}$ such that

$$
x^{*} \cdot x=\|x\| .
$$

More generally, for a given differentiable function $f: \mathbb{R}^{n} \rightarrow \mathbb{R}$, its gradient with respect to the norm $\|\cdot\|$ lives in $\left(\mathbb{R}^{n}\right)^{*}$ and its $\|\cdot\|_{*}$ norm corresponds to the norm of the derivative of $f$ as a linear map.

We set, for $p \in(1, n)$, and for $y=(t, x) \in \mathbb{R}_{+}^{n}=\mathbb{R}_{+} \times \mathbb{R}^{n-1}$,

$$
\bar{f}(y)=C_{n}(p)\|y-e\|^{\frac{p-n}{p-1}}
$$

where $e=(-1,0)$ and

$$
C_{n}(p)=\left(\frac{n}{(p-1) I_{n}(p)}\right)^{\frac{1}{p}-\frac{1}{n}}, \quad I_{n}(p)=\int_{\mathbb{R}^{n-1}}\|(1, x)\|^{-n q} d x .
$$

This normalization makes the function $\bar{f}^{\frac{n p}{n-p}}$ to be a probability density. Let us remark that it is sufficient to prove that $\bar{f}$ is extremal in (8), in order to get that all the functions given by $(9)$ are also extremals by the scaling invariance. The key point will be that $\bar{f}$ realizes the equality in (15) with

$$
f(y)=\bar{f}(y)^{\frac{n(p-1)}{n-p}}(y-e) \text { and } g(y)=-\nabla \bar{f}(y) .
$$

We shall proceed in 3 steps. Note that it is sufficient to prove (8) for non negative functions by taking $|f|$ in the inequality. We begin by establishing with the techniques of $[11,20]$ an inequality valid on two arbitrary smooth and compactly supported probability densities $F$ and $G$. Then, in a second part, we apply the inequality to $F=f^{\frac{n p}{n-p}}$ and $G=g^{\frac{n p}{n-p}}$, where $f$ and $g$ are still supposed to be non negative, smooth and compactly supported in $\mathbb{R}_{+}^{n}$. At this stage, we shall remove the assumptions by a density argument and the final step will then lead to the conclusion.

Consider $F$ and $G$ two smooth and compactly supported probability densities on $\mathbb{R}_{+}^{n}$, and let $\nabla \varphi$ the Brenier transportation map between $F(y) d y$ and $G(y) d y$. What follows is deeply inspired from [11, 20], and only differs by the treatment of the trace term. Then, we write

$$
\int_{\mathbb{R}_{+}^{n}} G(y)^{1-\frac{1}{n}} d y=\int_{\mathbb{R}_{+}^{n}} G(\nabla \varphi(y))^{-\frac{1}{n}} F(y) d y,
$$

by the definition of the Brenier map. Then, using the Monge-Ampère equation (14), we have

$$
\begin{aligned}
\int_{\mathbb{R}_{+}^{n}} G(y)^{1-\frac{1}{n}} d y & =\int_{\mathbb{R}_{+}^{n}}\left(\operatorname{Det} D^{2} \varphi(y)\right)^{\frac{1}{n}} F(y)^{1-\frac{1}{n}} d y, \\
& \leq \frac{1}{n} \int_{\mathbb{R}_{+}^{n}} \Delta \varphi(y) F(y)^{1-\frac{1}{n}} d y
\end{aligned}
$$

thanks to the arithmetic-geometric inequality. In the previous inequality, the Laplacian is understood as the absolutely continuous part of the distributional Laplacian. But, since $\varphi$ 
is convex, its distributional Hessian is a measure with values in the set of the semi-definite positive matrix. Then, it follows that

$$
\int_{\mathbb{R}_{+}^{n}} G(y)^{1-\frac{1}{n}} d y \leq \frac{1}{n} \int_{\mathbb{R}_{+}^{n}} \Delta_{\mathcal{D}^{\prime}} \varphi(y) F(y)^{1-\frac{1}{n}} d y .
$$

Let us introduce $\psi(y)=\varphi(y)-e \cdot y$. Then, $\varphi$ and $\psi$ share the same Laplacian. We have that $\nabla \varphi \in \mathrm{B} V_{\text {loc }}\left(\mathbb{R}_{+}^{n}\right)$, and since $F$ is smooth, we can use an integration by parts formula for BV functions. This leads to

$$
\int_{\mathbb{R}_{+}^{n}} G(y)^{1-\frac{1}{n}} d y \leq-\frac{1}{n} \int_{\mathbb{R}_{+}^{n}} \nabla \psi \cdot \nabla\left(F^{1-\frac{1}{n}}\right)(y) d y+\int_{\partial \mathbb{R}_{+}^{n}} F(y)^{1-\frac{1}{n}} \nabla \psi \cdot \mathbf{n},
$$

where $\mathbf{n}$ denotes the exterior normal vector, and in this case we have $\mathbf{n}=e$ at each point of the boundary. In addition, by the definition of the mass transportation, $\nabla \varphi(y) \in \mathbb{R}_{+}^{n}$, for all $y$, which exactly means that $\nabla \varphi$ satisfies $\nabla \varphi(y) \cdot \mathbf{n} \leq 0$. Since, $e \cdot e=1$, we get

$$
\int_{\partial \mathbb{R}_{+}^{n}} F(y)^{1-\frac{1}{n}} d y+\int_{\mathbb{R}_{+}^{n}} G(y)^{1-\frac{1}{n}} d y \leq-\frac{1}{n} \int_{\mathbb{R}_{+}^{n}}(\nabla \varphi(y)-e) \cdot \nabla\left(F^{1-\frac{1}{n}}\right)(y) d y .
$$

The next step consists in considering two non negative, smooth and still compactly supported functions $f$ and $g$ such that

$$
\int_{\mathbb{R}_{+}^{n}} f(y)^{\frac{n p}{n-p}} d y=\int_{\mathbb{R}_{+}^{n}} g(y)^{\frac{n p}{n-p}} d y=1,
$$

and setting $F=f^{\frac{n p}{n-p}}$ and $G=g^{\frac{n p}{n-p}}$. Then, (20) becomes

$$
\int_{\partial \mathbb{R}_{+}^{n}} f(y)^{\frac{(n-1) p}{n-p}} d y \leq-\frac{(n-1) p}{n(n-p)} \int_{\mathbb{R}_{+}^{n}} f(y)^{\frac{n(p-1)}{n-p}}(\nabla \varphi(y)-e) \cdot \nabla f(y) d y-\int_{\mathbb{R}_{+}^{n}} g(y)^{\frac{(n-1) p}{n-p}} d y .
$$

Using the definition of the dual norm and then applying Hölder inequality, it follows that

$$
\begin{gathered}
\int_{\partial \mathbb{R}_{+}^{n}} f(y)^{\frac{(n-1) p}{n-p}} d y \leq \frac{(n-1) p}{n(n-p)}\left(\int_{\mathbb{R}_{+}^{n}}\|\nabla \varphi(y)-e\|^{q} f(y)^{\frac{n p}{n-p}} d y\right)^{\frac{1}{q}}\left(\int_{\mathbb{R}_{+}^{n}}\|\nabla f(y)\|_{*}^{p} d y\right)^{\frac{1}{p}} \\
-\int_{\mathbb{R}_{+}^{n}} g(y)^{\frac{(n-1) p}{n-p}} d y
\end{gathered}
$$

and actually, by the definition of the transport map,

$$
\begin{gathered}
\int_{\partial \mathbb{R}_{+}^{n}} f(y)^{\frac{(n-1) p}{n-p}} d y \leq \frac{(n-1) p}{n(n-p)}\left(\int_{\mathbb{R}_{+}^{n}}\|y-e\|^{q} g(y)^{\frac{n p}{n-p}} d y\right)^{\frac{1}{q}}\left(\int_{\mathbb{R}_{+}^{n}}\|\nabla f(y)\|_{*}^{p} d y\right)^{\frac{1}{p}} \\
-\int_{\mathbb{R}_{+}^{n}} g(y)^{\frac{(n-1) p}{n-p}} d y
\end{gathered}
$$

At this stage, it is necessary to remove the compactness and smoothness hypothesis. Let us make two remarks. First, (21) has been establish only for compactly supported functions, but since the transport map $\nabla \varphi$ does not appear anymore, by density arguments, it remains valid as soon as each term is defined, typically for $f \in \dot{\mathrm{W}}^{1, p}\left(\mathbb{R}_{+}^{n}\right)$ and $g \in L^{p^{*}}\left(\mathbb{R}_{+}^{n}\right)$ (Indeed, the compactness hypothesis on $g$ was necessary to get sufficient regularity on $\nabla \varphi$ for the 
integration by parts). On the other hand, this beeing done, if we set $f=g=\bar{f}$, then, the transport map becomes the identity, so each step can be performed and provides an equality. As a conclusion for this step, (21) holds for any functions $f \in \dot{\mathrm{W}}^{1, p}\left(\mathbb{R}_{+}^{n}\right)$ and $g \in L^{p^{*}}\left(\mathbb{R}_{+}^{n}\right)$, and is an equality for $f=g=\bar{f}$.

Now, we can finish the proof. Let us set $g=\bar{f}$ in (21). This leads to, for all non negative $f$ in $\dot{\mathrm{W}}^{1, p}\left(\mathbb{R}_{+}^{n}\right)$ such that $\|f\|_{L^{p^{*}}\left(\mathbb{R}_{+}^{n}\right)}=1$,

$$
\int_{\mathbb{R}_{+}^{n}} f(y)^{\frac{(n-1) p}{n-p}} d y \leq A_{n}(p)\|\nabla f\|_{L^{p}\left(\mathbb{R}_{+}^{n}\right)}-B_{n}(p)
$$

with

$$
A_{n}(p)=\frac{(n-1) p}{n(n-p)}\left(\int_{\mathbb{R}_{+}^{n}}\|y-e\|^{q} \bar{f}(y)^{\frac{n p}{n-p}} d y\right)^{\frac{1}{q}}=\frac{(n-1) p}{n(n-p)}\left(\frac{p-1}{n-p}\right)^{\frac{1}{q}} C_{n}(p)^{\frac{n(p-1)}{n-p}} J_{n}(p)^{\frac{1}{q}}
$$

and

$$
B_{n}(p)=\int_{\mathbb{R}_{+}^{n}} \bar{f}(y)^{\frac{(n-1) p}{n-p}} d y=\frac{p-1}{n-p} C_{n}(p)^{\frac{(n-1) p}{n-p}} J_{n}(p),
$$

where

$$
J_{n}(p)=\int_{\mathbb{R}^{n-1}}\|(1, x)\|^{-q(n-1)} d x .
$$

Here, we remove the normalization. For all $f \in \dot{\mathrm{W}}^{1, p}\left(\mathbb{R}_{+}^{n}\right)$, the inequality (22) reads

$$
K(f)^{\frac{(n-1) p}{n-p}} Q(f) \leq A_{n}(p) K(f)-B_{n}(p),
$$

where we set

$$
K(f)=\frac{\|\nabla f\|_{L^{p}\left(\mathbb{R}_{+}^{n}\right)}}{\|f\|_{L^{p^{*}}\left(\mathbb{R}_{+}^{n}\right)}}, \text { and } Q(f)=\frac{\|f\|_{L^{\tilde{p}}\left(\partial \mathbb{R}_{+}^{n}\right)}^{\tilde{p}}}{\|\nabla f\|_{L^{p}\left(\mathbb{R}_{+}^{n}\right)}^{\tilde{p}}}
$$

hence

$$
Q(f) \leq K(f)^{-\frac{(n-1) p}{n-p}}\left(A_{n}(p) K(f)-B_{n}(p)\right) .
$$

The function $H: k \mapsto k^{-\frac{(n-1) p}{n-p}}\left(A_{n}(p) k-B_{n}(p)\right)$ achieves its maximum on $\mathbb{R}_{+}$at the point

$$
\begin{aligned}
k_{0} & =\frac{p(n-1)}{n(p-1)} \frac{B_{n}(p)}{A_{n}(p)} \\
& =\left(\frac{n-p}{p-1}\right)^{\frac{1}{q}} C_{n}(p) J_{n}(p)^{\frac{1}{p}} \\
& =\left(\int_{\mathbb{R}_{+}^{n}}\|\nabla \bar{f}\|_{*}^{p}\right)^{\frac{1}{p}}=K(\bar{f}),
\end{aligned}
$$

by a simple computation. As a conclusion, for all $f \in \dot{\mathrm{W}}^{1, p}\left(\mathbb{R}_{+}^{n}\right)$,

$$
Q(f) \leq H\left(k_{0}\right),
$$

with equality for $f=\bar{f}$, which ends the proof. 


\section{Further comments}

A natural question arising here concerns the identification of all the minimizers in (8). Following [11], the guess would be that, up to dilations and multiplication by constants, the function given by (9) are the unique minimizers in (8). Unfortunately, it supposes that we are able to perform the integration by parts (19), only assuming that $f \in \dot{\mathrm{W}}^{1, p}\left(\mathbb{R}_{+}^{n}\right)$, which is not really a problem, but also $g \in L^{p^{*}}\left(\mathbb{R}_{+}^{n}\right)$ not necessarily compactly supported. In that case, the normal derivative of $\varphi$ has no reason even to exist on the boundary, so, though the problem seems to be only technical, we left it open.

Another interesting remark is the following. If we don't introduce the vector $e$ in (19), then the trace term does not appear anymore, and, replacing $\bar{f}$ by the minimizers in the corresponding sharp Sobolev inequality in $\mathbb{R}^{n}$, we can end the proof in the same way as in [11] and get the Sobolev inequality (1) for $\mathbb{R}_{+}^{n}$, which means that $\mathbb{R}_{+}^{n}$ is a gradient domain in the sense of [20] (This was already proved in [19]). It is not the only one, and a natural criterion can be directly derived from the proof. Indeed, it is easy to see that, if $\Omega \subset \mathbb{R}^{n}$ satisfies that there exists some $y_{0} \in \Omega$ such that, $\forall y \in \Omega$ and $x \in \partial \Omega$,

$$
\left(y-y_{0}\right) \cdot \mathbf{n}_{x} \leq 0 \text {, and }\left(x-y_{0}\right) \cdot \mathbf{n}_{x}=0,
$$

where $\mathbf{n}$ stands for the exterior normal of the boundary at the point $x$, then $\Omega$ is a gradient domain, and the minimizers takes the form

$$
f(y)=\left(\left\|y-y_{0}\right\|^{q}+\sigma^{q}\right)^{1-\frac{p}{n}}, \quad \sigma>0 .
$$

In particular, this is the case for the following conical subsets of $\mathbb{R}_{+}^{n}$,

$$
\Omega=\left\{(t, x) \in \mathbb{R}_{+}^{n} ; F\left(\frac{x}{t}\right) \leq 0\right\},
$$

for any convex function $F: \mathbb{R}^{n-1} \rightarrow \mathbb{R}$.

\section{References}

[1] Aguen, M., Ghoussoub, N., Kang, X., Geometric inequalities via a general comparison principle for interacting gases, Geom. Funct. Anal., 14(1), (2004), 215-244.

[2] Aubin, T., Problèmes isopérimétriques et espaces de Sobolev, J. Differential Geometry, 11, 4, (1976), 573-598.

[3] Barthe, F., On a reverse form of the Brascamp-Lieb inequality, Invent. Math., 134(2), (1998), 335-361.

[4] Beckner, W., Sharp Sobolev inequalities on the sphere and the Moser-Trudinger inequality, Annals of Mathematics, 138 (1993), 213-242.

[5] Brenier, Y., Polar factorization and monotone rearrangment of vector-valued functions, Comm. Pure Appl. Math. 44(4), (1991), 375- 417.

[6] BrÉzis, H., Lieb, E., Sobolev inequalities with a remainder term, J. Funct. Anal. 62, (1985), 73-86.

[7] Caffarelli, L.A., Boundary regularity of maps with convex potentials, Comm. Pure Appl. Math., 45(9), (1992), 1141-1151. 
[8] Caffarelli, L.A., The regularity of mappings with a convex potential, J. Amer. Math. Soc., 5(1), (1992), 99-104.

[9] Caffarelli, L.A., Boundary regularity of maps with convex potentials II, Ann. Math. (2), 144(3), (1996), 453-496.

[10] Carlen, E.A., Loss, M., Extremals of functionals with competing symmetries, J. Funct. Anal., 88(2), (1990), 437-456.

[11] Cordero-Erausquin, D., Nazaret, B., Villani, C., A mass-transportation approach to sharp Sobolev and Gagliardo-Nirenberg inequalities, Advances in Mathematics, 182 (2004), 307-332.

[12] Del Pino, M., Dolbeault, J., Best constants for Gagliardo-Nirenberg inequalities and application to nonlinear diffusions, J. Math. Pures Appl., 81(9), (2002), 847-875.

[13] Demengel, F., Hebey, E., On some nonlinear equations involving the $p$-Laplacian with critical Sobolev growth, Adv. Differential Eq., 3(4), (1998), 533-574.

[14] Demengel F., Nazaret B., On some nonlinear partial differential equations involving the $p$-Laplacian and critical Sobolev trace maps, Asympt. Anal., 23(2), (2000), 135-156.

[15] Escobar, J.F., Sharp constant in a Sobolev trace inequality, Indiana University Mathematics Journal, 37 (1988), 687-698.

[16] Gidas, B., Ni, W., Nirenberg, L., Symmetry and related properties via the maximum principle, Comm. Math. Phys., 68, (1979), 209-243.

[17] Guedda, M., Véron, L., Quasilinear elliptic equations involving critical Sobolev exponents, Nonlinear Analysis, 13(8), (1989), 879-902.

[18] Humbert, E., Optimal trace Nash inequality, Geom. Funct. Anal., 11(4), (2001), 759772.

[19] Lions, P.L., The concentration-compactness principle in the calculus of variations. The locally compact case II, Rev. Mat. Iberoamericana, 1(2), (1985), 45-121.

[20] Maggi, F., Villani, C., Balls have the worst best Sobolev inequalities. To appear in J. Geom. Anal.

[21] McCann, R.J., Existence and uniqueness of monotone measure-preserving maps, Duke Math. J., 80(2), (1995), 309-323.

[22] Milman, V.D., Schechtman, G., Asymptotic Theory of Finite-Dimensional NormedSpaces, Springer, Berlin, 1986 (with an appendix by M. Gromov).

[23] Lieb, E., Loss, M., Analysis, second ed. American Mathematical Society, Providence, RI, 2001.

[24] Talenti, G., Best constants in Sobolev inequality, Ann. Mat. Pura Appl. (IV), 110, (1976), 353-372.

[25] Villani, C., Topics in Mass Transportation, Vol. 58 of Graduate Studies in Mathematics. American Mathematical Society, Providence, RI, 2003. 